\title{
Symmetries and Lagrangian time-discretizations of Euler equations
}

\author{
Alexei V. Penskoi*
}

\begin{abstract}
In the late 80s - early 90s J. Moser and A. P. Veselov considered Lagrangian discrete systems on Lie groups with additional symmetry conditions imposed on Lagrangians. They observed that such systems are often integrable time-discretizations of integrable Euler equations on these Lie groups. In recent papers we studied Lagrangian discrete systems with additional symmetry requirements on certain infinitedimensional Lie groups. We will discuss some interesting properties of these systems.

2000 Mathematical Subject Classification 34K99, 22E65, 70H99

Keywords: Euler equation, Discrete Lagrangian system, Virasoro group, Camassa-Holm equation, Korteweg-de Vries equation, HunterSaxton equation.
\end{abstract}

\section{Introduction}

The goal of this talk is to review some recent results in discrete Lagrangian systems [1, 2, 3, 4].

Let $M$ be a manifold and $L$ be a function on $M \times M$. The discrete Lagrangian system with the Lagrangian $L$ is the system of difference equations

$$
\delta S=0,
$$

which describes the stationary points of the functional $S=S(X)$, defined on the space of sequences $X=\left(x_{k}\right), x_{k} \in M, k \in \mathbb{Z}$ by a

*Centre de recherches mathématiques, Université de Montréal, C. P. 6128, succ. Centreville, Montréal, Québec, H3C 3J7, Canada e-mail: penskoi@crm.umontreal.ca 
formal sum

$$
S=\sum_{k \in \mathbb{Z}} L\left(x_{k}, x_{k+1}\right) .
$$

Sometimes this system has a continuous limit; in that case it is called a discrete version of the corresponding system of differential equations.

For integrable equations it is natural to ask for discretizations which are also integrable. The theory of integrable Lagrangian discretizations of classical integrable systems was initiated by Moser and Veselov [5, 6].

Let us assume that $M$ is a Lie group $G$. It was observed by Veselov and Moser [5, 6, 7, 8, that symmetric

$$
L(x, y)=L(y, x)
$$

and right-invariant (or left-invariant)

$$
\forall g \in G \quad L(x g, y g)=L(x, y)
$$

Lagrangians often correspond to integrable systems which are discretizations of the Euler equations corresponding to some rightinvariant (or left-invariant) metrics on $G$.

In particular, it was shown that the discrete Lagrangian system on the orthogonal group $O(n)$ with the Lagrangian $L(X, Y)=$ $\operatorname{tr}\left(X J Y^{T}\right)$, where $X, Y \in O(n)$ and $J$ is a symmetric positive-defined matrix, can be considered as an integrable discrete version of the Euler-Arnold top [9].

The first attempt to generalize this approach to the infinitedimensional situation was done in [7, where the case of the group of area-preserving plane diffeomorphisms $\operatorname{SDiff}\left(\mathbb{R}^{2}\right)$ was considered.

This paper was followed by recent papers [1, 2, 3, 4, where discrete Lagrangian systems on the Virasoro group have been discussed. The interest in the Virasoro group was motivated by the important observation due to Khesin and Ovsienko [10] that the Korteweg-de Vries equation can be interpreted as an Euler equation on the Virasoro algebra. This result was later generalized by Misiołek [11] and Khesin [12]. They remarked that the Euler equation corresponding to the rightinvariant $H_{\alpha, \beta}^{1}$-metric on the Virasoro group has the form

$$
\alpha\left(v_{t}+3 v v_{x}\right)-\beta\left(v_{x x t}+2 v_{x} v_{x x}+v v_{x x x}\right)-b v_{x x x}=0 .
$$

This is a three-parameter family of integrable equation including the Camassa-Holm, the Hunter-Saxton and the Korteweg-de Vries equations. 
The plan of the talk is following. We shall start by recalling the basic facts about the Euler equation in Section 2 and the Virasoro group in Section 3 Then we will expose in Section 4 results of Ovsienko, Khesin and Misiołek [10, 11, 12] concerning Euler equations on the Virasoro group. Section 5 is devoted to discrete Lagrangian systems, and the final Section [6] contains results on discrete Lagrangian systems on the Virasoro group.

\section{The Euler equation}

Let $G$ be a Lie group, $\mathfrak{g}$ its Lie algebra. A right-invariant metric on $G$ is completely defined by its restriction to $\mathfrak{g}$

$$
(,): \mathfrak{g} \times \mathfrak{g} \rightarrow \mathbb{R} .
$$

This scalar product on $\mathfrak{g}$ defines a linear map $A: \mathfrak{g} \longrightarrow \mathfrak{g}^{*}$ such that

$$
(\xi, \eta)=\langle A \xi, \eta\rangle
$$

where $\xi, \eta \in \mathfrak{g}$ and $\langle$,$\rangle denotes the natural pairing between \mathfrak{g}$ and $\mathfrak{g}^{*}$. The operator $A$ is called the inertia operator.

To describe a geodesic $g(t)$ on $G$ corresponding to (, ), we transport its velocity vector to the identity by the right translation

$$
v(t)=R_{g^{-1}(t) *} \frac{d}{d t} g(t) .
$$

Since $v(t)$ is an element of $\mathfrak{g}$, we can consider $m=A v$ which is an element of the dual space $\mathfrak{g}^{*}$. Then $m$ satisfies the Euler equation given by the following explicit formula:

$$
\frac{d m}{d t}=-a d_{A^{-1} m}^{*} m \text {. }
$$

This is a standard result which can be found in 9]. If we start with a left-invariant metric, the sign in (11) is reversed. Since $A$ is nondegenerate, one can rewrite the Euler equation in terms of $v$ on the Lie algebra $\mathfrak{g}$.

Example 1. 9 Let us consider $S O(N)$. We identify the dual space $\mathfrak{s o}(N)^{*}$ with the algebra $\mathfrak{s o}(N)$ using the standard product $\operatorname{tr} X Y^{T}$ in the space of $N \times N$ matrices. Let $J$ be a symmetric positive-definite matrix. The inertia operator defined as

$$
A \Omega=J \Omega+\Omega J, \quad \Omega \in \mathfrak{s o}(N)
$$


gives the Euler equation on $S O(N)$. It can be written in a more familiar form as a system

$$
\left\{\begin{array}{l}
\dot{M}=[M, \Omega] \\
M=J \Omega+\Omega J
\end{array}\right.
$$

This is the Euler-Arnold equation for the $N$-dimensional rigid body.

Example 2. 9] Let us consider the group $\operatorname{SDiff}\left(\mathbb{R}^{2}\right)$ of volumepreserving diffeomorphisms of $\mathbb{R}^{2}$. The corresponding Lie algebra is the algebra of all vector fields with divergence 0 on $\mathbb{R}^{2}$. Let us consider the scalar product of two such vector fields defined as

$$
\left(v_{1}, v_{2}\right)=\iint_{\mathbb{R}^{2}} v_{1} \cdot v_{2} d x_{1} d x_{2},
$$

where $\cdot$ denotes the standard scalar product of vectors in $\mathbb{R}^{2}$. We can find the Euler equation corresponding to this scalar product. We will write it on the Lie algebra in the following form:

$$
\left\{\begin{aligned}
\frac{\partial v}{\partial t}+v \nabla v & =-\operatorname{grad} p \\
\operatorname{div} v & =0 .
\end{aligned}\right.
$$

This is the two-dimensional Euler equation for the ideal fluid. Here $p$ is a pressure, it is some unknown function which plays the role of a Lagrangian multiplier defined by the constraint $\operatorname{div} v=0$.

\section{The Virasoro group and the Vira- soro algebra}

Let Diff $+\left(S^{1}\right)$ be the group of diffeomorphisms of $S^{1}$ preserving the orientation. We shall represent an element of $\operatorname{Diff}_{+}\left(S^{1}\right)$ as a diffeomorphism $f: \mathbb{R} \rightarrow \mathbb{R}$ such that

1. $f \in C^{\infty}(\mathbb{R})$,

2. $f^{\prime}(x)>0$,

3. $f(x+2 \pi)=f(x)+2 \pi$.

Such a representation is not unique. Indeed, the functions $f+2 \pi k, k \in$ $\mathbb{Z}$ represent one element of $\operatorname{Diff}_{+}\left(S^{1}\right)$.

There exists a non-trivial central extension of Diff ${ }_{+}\left(S^{1}\right)$ which is unique up to an isomorphism 
This extension is called the Virasoro group (or the Bott-Virasoro group) and is denoted by Vir. Elements of Vir are pairs $(f, F)$, where $f \in \operatorname{Diff}_{+}\left(S^{1}\right), F \in \mathbb{R}$. The product of two elements is defined with the help of the Bott cocycle as

$$
(f, F) \circ(g, G)=\left(f \circ g, F+G+\int_{0}^{2 \pi} \log (f \circ g)^{\prime} d \log g^{\prime}\right) .
$$

The unit element of $\operatorname{Vir}$ is $(i d, 0)$. The inverse element of $(f, F)$ is $\left(f^{-1},-F\right)$.

The Virasoro algebra vir is a Lie algebra corresponding to the Virasoro group. It is the central extension of the algebra vect $\left(S^{1}\right)$ of vector fields on the circle $S^{1}$

$$
\operatorname{vir}=\operatorname{vect}\left(S^{1}\right) \oplus \mathbb{R} .
$$

We represent an element of $\operatorname{vect}\left(S^{1}\right)$ as $v(x) \partial_{x}$, where $v$ is a $2 \pi$-periodic function. Thus an element of the Virasoro algebra is a pair $\left(v(x) \partial_{x}, a\right)$. The algebra commutator in vir is defined with the help of the GelfandFuchs cocycle as

$$
\left[\left(v(x) \partial_{x}, a\right],\left[w(x) \partial_{x}, b\right]\right)=\left(\left(-v w_{x}+v_{x} w\right)(x) \partial_{x}, \int_{0}^{2 \pi} v_{x x x} w d x\right)
$$

\section{The Euler equation for the $H_{\alpha, \beta^{-}}^{1}$ metric on the Virasoro group}

In 1987 Khesin and Ovsienko remarked [10] that the Kortewegde Vries equation can be interpreted as an Euler equation on the Virasoro group. This result was later generalized by Misiołek [11] and Khesin [12] in the following way.

Let $\alpha$ and $\beta$ be two non-negative real numbers such that $\alpha^{2}+\beta^{2} \neq$ 0 . Let us define the $H_{\alpha, \beta}^{1}$-metric on the Virasoro algebra by the formula

$$
\left(\left(v(x) \partial_{x}, a\right),\left(w(x) \partial_{x}, b\right)\right)_{H_{\alpha, \beta}^{1}}=\int_{0}^{2 \pi}\left(\alpha v(x) w(x)+\beta v_{x}(x) w_{x}(x)\right) d x+a b .
$$


Theorem 1 The Euler equation corresponding to the right-invariant $H_{\alpha, \beta}^{1}$-metric on the Virasoro group has the form

$$
\begin{gathered}
\alpha\left(v_{t}+3 v v_{x}\right)-\beta\left(v_{x x t}+2 v_{x} v_{x x}+v v_{x x x}\right)-b v_{x x x}=0, \\
b_{t}=0 .
\end{gathered}
$$

Since $b$ is a constant, we can consider (2) as an equation for $v$ depending on three constants $\alpha, \beta, b$. We obtain a three-parametric family of integrable equations which we call the Camassa-Holm family. The reason for this name is the following. We have a freedom of multiplication of the equation by a non-zero constant, the twodimensional scaling symmetry group $v \rightarrow \lambda v, t \rightarrow \mu t, x \rightarrow \lambda \mu x$ and the Galilean group $v \rightarrow v+c, x \rightarrow x+d t, t \rightarrow t$. Modulo these symmetries we have just one generic orbit, containing the equation with $\alpha=1, \beta=1, b=0$ :

$$
v_{t}-v_{x x t}+3 v v_{x}-2 v_{x} v_{x x}-v v_{x x x}=0,
$$

which is one of the canonical forms of the Camassa-Holm shallowwater equation 13

$$
v_{t}+2 \kappa v_{x}+\gamma v_{x x x}-v_{x x t}+3 v v_{x}-2 v_{x} v_{x x}-v v_{x x x}=0 .
$$

We have also four degenerate orbits. When $\alpha \neq 0, \beta=0, b \neq 0$ the equation (2) is equivalent to the $\mathrm{KdV}$ equation:

$$
v_{t}+3 v v_{x}+v_{x x x}=0 .
$$

Further degeneration $\alpha \neq 0, \beta=0, b=0$ leads to the dispersionless $\mathrm{KdV}$ equation (sometimes also called the Hopf equation):

$$
v_{t}+3 v v_{x}=0 .
$$

When $\alpha=0, \beta \neq 0$ we have the Hunter-Saxton equation [14]

$$
v_{x x t}+2 v_{x} v_{x x}+v v_{x x x}=0 .
$$

Finally if both $\alpha$ and $\beta$ are zero (but $b$ is not) we simply have

$$
v_{x x x}=0 .
$$




\section{$5 \quad$ Discrete Lagrangian systems}

The definition of a discrete Lagrangian system is already done in Introduction. Also, we have already mentioned in Introduction the observation by Veselov and Moser that symmetric and right-invariant (or left-invariant) Lagrangians often correspond to integrable systems which are discretizations of the Euler equations corresponding to some right-invariant (or left-invariant) metrics on $G$. Let us consider some examples.

Example 3. 6] Let us consider the group $S O(N)$. Let $J$ be a symmetric positive-definite matrix. The Lagrangian

$$
L(X, Y)=\operatorname{tr}\left(X J Y^{T}\right)
$$

is left-invariant and symmetric. Let us introduce the discrete angular velocity $\omega_{k}=X_{k}^{T} X_{k-1}$ and the discrete angular momentum $M_{k}=$ $\omega_{k}^{T} J-J \omega_{k}$. The discrete Euler-Lagrange equation $\frac{\delta S}{\delta X_{k}}=0$ can be written in the following form (discrete Euler-Arnold equation).

$$
\left\{\begin{aligned}
M_{k+1} & =\omega_{k} M_{k} \omega_{k}^{-1}, \\
M_{k} & =\omega_{k}^{T} J-J \omega_{k} .
\end{aligned}\right.
$$

This equation is integrable and its continuous limit is the Euler-Arnold equation of $N$-dimensional rigid body considered in the Example 1.

Example 4. 7, 15] Let us consider the group $\operatorname{SDiff}\left(\mathbb{R}^{2}\right)$. The Lagrangian

$$
L(f, g)=\iint_{\mathbb{R}^{2}} \operatorname{tr}\left(J(f) J(g)^{-1}\right) d x_{1} d x_{2},
$$

where $f, g \in \operatorname{SDiff}\left(\mathbb{R}^{2}\right)$ and $J$ is the Jacobian, is right-invariant and symmetric. Let $\varphi=f_{k} \circ f_{k-1}^{-1}, \psi=f_{k+1} \circ f_{k}^{-1}$ and $\chi=\varphi^{-1}$. The discrete Euler-Lagrange equation $\frac{\delta S}{\delta f_{k}}=0$ can be written in the following form.

$$
\left\{\begin{aligned}
\psi_{1} & =\tau x_{1}+a_{1}-\chi_{1} \\
\psi_{2} & =\tau x_{2}+a_{2}-\chi_{2} \\
\operatorname{tr} J(\varphi) & =\tau .
\end{aligned}\right.
$$

In these equations $\tau, a_{1}, a_{2}$ are constants, $\psi_{i}, i=1,2$ are components of $\psi=\left(\psi_{1}, \psi_{2}\right): \mathbb{R}^{2} \rightarrow \mathbb{R}^{2}$, and $\chi_{i}, i=1,2$ are defined in the analogous way. This discrete system is integrable. If $\tau=2$ one can find the continuous limit

$$
\left\{\begin{aligned}
v_{t}+v \nabla v & =0 \\
\operatorname{div} v & =0
\end{aligned}\right.
$$


This is an equation of the isobaric flow, a particular case of the twodimensional Euler equation for the ideal fluid when the pressure is equal to zero.

\section{Discrete Lagrangian systems on the Virasoro group}

In this section we present some results of papers [1, 2, 3, 4. Let us consider discrete Lagrangian systems on the Virasoro group. It was explained in Section [5 that it is natural to consider right-invariant symmetric Lagrangians.

We shall use the following simple observation to construct Lagrangians. Let us consider a Lagrangian $L(x, y)$ on a Lie group $G$. Let $H(x)=L(x, e)$, where $e$ is the identity element of $G$. A rightinvariant Lagrangian $L(x, y)$ is completely determined by $H$. Indeed, $L(x, y)=L\left(x y^{-1}, e\right)=H\left(x y^{-1}\right)$.

Let us now consider a right-invariant symmetric Lagrangian. It is easy to see that symmetric Lagrangian corresponds to inverseinvariant function $H$ :

$$
H\left(x^{-1}\right)=H(x) .
$$

Let us start by considering the discrete Lagrangian systems on Vir corresponding to the functions $H$ of the following form [4]

$$
H((f, F))=F^{2}+\int_{0}^{2 \pi} V\left(f(x)-x, f^{\prime}(x)\right) d x,
$$

where $f$ is a diffeomorphism, $F \in \mathbb{R}$, and $V\left(x_{1}, x_{2}\right)$ is an arbitrary, $2 \pi$ periodic in $x_{1}$ function of two variables, which satisfies the condition:

$$
V_{1}(0,1)=0 .
$$

Let us explain a motivation for such a choice of $H$. We are interested in Lagrangian time-discretizations of the Camassa-Holm family. This family corresponds to $H_{\alpha, \beta}^{1}$-metrics, and these metrics depend on

elements of the algebra and their first derivatives. So it is natural to consider a function $H$ of the form

$$
H((f, F))=F^{2}+\int_{0}^{2 \pi} U\left(f(x), f^{\prime}(x), x\right) d x,
$$


where $U\left(x_{1}, x_{2}, x_{3}\right)$ is an arbitrary function that is $2 \pi$-periodic with respect to the first argument. Periodicity of $V$ or $U$ is related to the fact that $f(x)$ and $f(x)+2 \pi$ represent the same diffeomorphism of $S^{1}$. However, it turns out that the continuous limit of the system corresponding to (4) is, in general, some quite boring ODE having nothing in common with Euler equations. To obtain something interesting, it is necessary to impose the additional conditions that $U$ has the form $U\left(x_{1}, x_{2}, x_{3}\right)=V\left(x_{1}-x_{3}, x_{2}\right)$ and $V_{1}(0,1)=0$. In this case the continuous limit is some equation from the Camassa-Holm family.

The form (3) is very general since the difference $f(x)-x$, which is a $2 \pi$-periodic function, is as natural as $f(x)$ itself. The only property which might look artificial is the last condition on the partial derivative of $V$. See [4 for a detailed discussion.

In general, the Lagrangian corresponding to (3) is not symmetric. Hence, it is not expected to give us an integrable system. Nevertheless, it is interesting to look at such Lagrangians because of the following interesting phenomenon. In general, if we consider a Lie group $G$, a continuous limit of a right-invariant discrete Lagrangian system is a geodesic flow corresponding to some right-invariant metric. This geodesic flow is, in general, not integrable. Let us for example look at the case of $S O(N)$. It is known that for $N>3$ a geodesic flow of a general right-invariant metric is non-integrable (see e.g. [16]). Hence, the result on the Virasoro group turns out to be surprising: in spite of the fact that the class of discrete systems we consider (3) is quite general, in the continuous limit we have the family of integrable equations. There are integrable cases of the Euler equations on $S O(N)$ (for example the Manakov metrics [17) but no analogues of the result for the Virasoro group are known for them.

The heuristic explanation of this phenomenon is that the equations of the Camassa-Holm family can be considered as nonlinear analogues of the harmonic oscillators on the Virasoro group: in the first approximation all Hamiltonian systems near equilibriums behave like harmonic oscillators. Thus, in some sense this demonstrates a universal nature of the Camassa-Holm family of equations. See Discussion in [4] for more details.

Let us now consider the question of the integrability of discrete Lagrangian systems on the Virasoro group. Unfortunately we do not know examples of such systems, but we have good candidates for integrability. A good candidate to be an integrable discretization of the $\mathrm{KdV}$ equation was considered in [2], but it is the case of the discretiza- 
tion of the Hunter-Saxton equation [3] which is most promising. Let us consider this case.

The Hunter-Saxton equation corresponds to the Euler equation for the $H_{0,1}^{1}$-metric. This metric depends only on first derivatives of algebra elements. Hence, it is natural to look at such functions $H$ (3) that $V$ depends only on $f^{\prime}$. As it was explained before, it is natural to study right-invariant symmetric Lagrangians when looking for integrable systems, and symmetric Lagrangians correspond to inverseinvariant functions $H$.

It can be easily verified that functions $H$ of the form

$$
H((f, F))=F^{2}+\int_{0}^{2 \pi} V\left(f^{\prime}(x)\right) d x
$$

are inverse-invariant when the function $V$ satisfies the condition

$$
x V\left(\frac{1}{x}\right)=V(x)
$$

The simplest function $V$ satisfying the property (6) is $V(x)=\sqrt{x}$. Let us consider this case.

We have $V(x)=\sqrt{x}$. The function $V$ defines $H$ as described in (5). The function $H$ defines a Lagrangian $L$ as described above:

$$
L\left(\left(f_{k}, F_{k}\right),\left(f_{k+1}, F_{k+1}\right)\right)=H\left(\left(f_{k}, F_{k}\right) \circ\left(f_{k+1}, F_{k+1}\right)^{-1}\right) .
$$

Hence we are considering a functional

$$
S=\sum_{k \in \mathbb{Z}} L\left(\left(f_{k}, F_{k}\right),\left(f_{k+1}, F_{k+1}\right)\right)
$$

where $\left\{\left(f_{k}, F_{k}\right)\right\}$ is a sequence of points on Vir. We can find the EulerLagrange equations $\frac{\delta S}{\delta\left(f_{k}, F_{k}\right)}=0$. They are [3]

$$
\begin{gathered}
-\Omega_{k}+\Omega_{k+1}=0, \\
{\left[-2 \Omega_{k}\left(\log \left(\left(\omega_{k}\right)^{\prime}\right)\right)^{\prime}-\frac{1}{2} \sqrt{\left(\omega_{k}\right)^{\prime}}+\right.} \\
\left.+2 \Omega_{k+1}\left(\log \left(\left(\omega_{k+1}^{-1}\right)^{\prime}\right)\right)^{\prime}-\frac{1}{2} \sqrt{\left(\omega_{k+1}^{-1}\right)^{\prime}}\right]^{\prime}=0,
\end{gathered}
$$


where $\left(\omega_{k}, \Omega_{k}\right)$ and $\left(\omega_{k+1}, \Omega_{k+1}\right)$ are discrete analogues of angular velocities,

$$
\left(\omega_{l}, \Omega_{l}\right)=\left(f_{l-1}, F_{l-1}\right) \circ\left(f_{l}, F_{l}\right)^{-1}, \quad l \in \mathbb{Z} .
$$

The equation (17) is just saying that $\Omega_{k}$ is an integral, $\Omega_{k}=\Omega$. As for (8), we can integrate this equation once and put

$$
\Phi=\frac{1}{\sqrt{\left(\omega_{k}\right)^{\prime}}}, \quad \Psi=\frac{1}{\sqrt{\left(\omega_{k+1}^{-1}\right)^{\prime}}} .
$$

We obtain the equation

$$
8 \Omega\left(-\frac{\Phi^{\prime}}{\Phi}+\frac{\Psi^{\prime}}{\Psi}\right)+\frac{1}{\Phi}+\frac{1}{\Psi}+C=0
$$

where $C$ is a constant of integration. This equation is equivalent to the equation

$$
\Psi^{\prime}+\Psi\left(\frac{C}{8 \Omega}+\frac{1}{8 \Omega \Phi}-\frac{\Phi^{\prime}}{\Phi}\right)+\frac{1}{8 \Omega}=0
$$

This is a linear first-order differential equation for $\Psi$ with periodic coefficients depending on $\Phi$. For generic $\Phi$ it has only one solution, so $\Psi$ is determined by $\Phi$ up to a constant $C$. Reconstructing $\omega_{k+1}^{-1}$ from $\Psi$, we obtain another constant, so we have a following result: $\omega_{k+1}$ is obtained from $\omega_{k}$ by a two-parametric correspondence. To find $\omega_{k+1}$ starting from $\omega_{k}$, we must solve a first-order linear differential equation to find $\omega_{k+1}^{-1}$, and then reconstruct $\omega_{k+1}$ from $\omega_{k+1}^{-1}$ by inversion. The analogous situation was observed in the case of $\operatorname{SDiff}\left(\mathbb{R}^{2}\right)[7$ which is integrable [15. For this reason we consider system (78) to be a good candidate for an integrable Lagrangian discretization of the HunterSaxton equation. See [3] for more detailed discussion.

It should be remarked, that one can obtain the Hunter-Saxton equation as an Euler equation not only on the Virasoro group, but also on the group of orientation-preserving diffeomorphisms of the circle. This leads to a particularly simple discretization

$$
\left[\sqrt{\left(\omega_{k}\right)^{\prime}}+\sqrt{\left(\omega_{k+1}^{-1}\right)^{\prime}}\right]^{\prime}=0,
$$

which also has some nice properties, see [3] for details. 


\section{Acknowledgments}

The author is very grateful to the Organizing Committee of the Workshop on Superintegrability in Classical and Quantum Systems for giving him the possibility to give this talk.

\section{References}

[1] A. V. Penskoi Discrete Lagrangian systems on the Virasoro group. Vestnik Moskov. Univ. Ser. I Math. Mekh. (1996) no 4 99-102 (Russian), English translation in Moscow Univ. Math. Bull. 51 (1996) no 4 52-54.

[2] A. V. Penskoi Lagrangian time-discretization of the Kortewegde Vries equation Phys. Lett. A (2000) V. 269 no. 4 224-229.

[3] A. V. Penskoi Lagrangian time-discretization of the HunterSaxton equation. To appear in Phys. Lett. A.

[4] A. V. Penskoi, A. P. Veselov Discrete Lagrangian systems on the Virasoro group and Camassa-Holm family. Submitted to Nonlinearity.

[5] A. P. Veselov, Integrable systems with discrete time, and difference operators Funkts. Anal. Pril. 1988 V. 22 no. 2 1-13 (Russian), Translation in Funct. Anal. Appl. 1988 V. 22 no. 2 83-93.

[6] J. Moser, A. P. Veselov, Discrete versions of some classical integrable systems and factorization of matrix polynomials Comm. Math. Phys. 1991 V. 139 no. 2 217-243.

[7] J. Moser, A. P. Veselov, Two-dimensional "discrete hydrodynamics" and Monge-Ampère equation Preprint of ETH, Zürich, 1993. Ergodic Theory and Dynamical Systems (2002), V. 22, 1575-1583.

[8] A. P. Veselov, Integrable Lagrangian relations and factorization of matrix polynomials Funkts. Anal. Pril. 1991 V. 25 no. 2 38-49 (Russian), Translation in Funct. Anal. Appl. 1991 V. 25 no. 2 112-122.

[9] V. I. Arnold Mathematical methods of classical mechanics Springer-Verlag, New York 1998.

[10] V. Yu. Ovsienko, B. A. Khesin The super Korteweg-de Vries equation as an Euler equation. Funkts. Anal. Pril. (1987) V. 21 no. 4 
81-82 (Russian), English translation in Funct. Anal. Appl. (1987) V. 21 no. 4 329-331.

[11] G. Misiołek A shallow water equation as a geodesic flow on the Bott-Virasoro group. J. Geom. Phys. (1998) V. 24 203-208.

[12] B. Khesin, G. Misiołek Euler equations on homogeneous spaces and Virasoro orbits. Preprint (2001) To appear in Adv. Math.

[13] R. Camassa, D. Holm An integrable shallow water equation with peaked solutions. Physica D (1993) V. 71 1661-1664.

[14] J. K. Hunter, R. Saxton Dynamics of director fields. SIAM Journal on Appl. Math. (1991) V. 51 no. 6 1498-1521

[15] E. V. Ferapontov, A. P. Veselov Appendix to [7].

[16] A.P. Veselov On the conditions of integrability of Euler equations on SO(4). Dokl. Akad. Nauk SSSR (1983) V. 270 no. 6. 12981300 (Russian). English translation in Soviet Math. Dokl. (1983) V. 27 no. 3 740-742.

[17] S.V. Manakov Note on the integration of Euler equations of the dynamics of an n-dimensional rigid body. Funkts. Anal. Pril. (1976) V. 10 no. 4 93-94 (Russian), English translation in Funct. Anal. Appl. (1976) V. 10 no. 4 328-329. 